E-017 METHOD OF INTRA-ARTERIAL ADMINISTRATION IN A TRANSIENT MCAO RAT MODEL TO MIMIC THE TREATMENT OF THE HUMAN THROMBECTOMY PATIENT

S Goodwin, J Roberts, K Pennypacker, J Fraser*. University of Kentucky, Lexington, KY

10.1136/neurintsurg-2020-SNIS.53

Endovascular thrombectomy is routinely performed in patients undergoing a large vessel occlusion. This process not only allows for the removal of a blood clot but also for the selective delivery of potential therapeutics directly to the site of injury. The intra-arterial (IA) route of drug administration in the mouse was developed to bridge the gap between animal stroke treatments and clinical stroke therapy. We have previously shown this delivery method targets the site of injury while blunting systemic effects that have proven problematic with intraperitoneal routes. Here, we adapted the IA method for use in rats, combining it with a clinically relevant large vessel occlusion stroke model (MCAO). Our goal was to characterize variances in the model in order to optimize potential therapeutic delivery methods to the ipsilaterally affected hemisphere. Male and female Sprague-Dawley rats (4 months of age) were subjected to placement of micro-angio tubing at the bifurcation of the common carotid artery (CCA), with delivery through the internal carotid artery (ICA). We then determined the optimal infusion rate and volume using injection of India ink and evaluation of vascular distribution within the brain and the liver. Importantly, we found the infusion rate and volume varied depending on sex and body weight. India ink was selectively delivered to the ipsilateral side of the brain in males $(370-460 \mathrm{~g})$ at $4 \mu \mathrm{l} / \mathrm{min}$ with a max volume of 25 $\mu \mathrm{l}$ and in females $(250-300 \mathrm{~g})$ at $2.5 \mu \mathrm{l} / \mathrm{min}$ with a $\max$ volume of $25 \mu \mathrm{l}$. Following these guidelines, no ink was observed in the liver of these animals, indicating reduced systemic circulation of administered compounds. We then performed a 5-hour transient MCAO, on male and female Sprague-Dawley rats (4 months of age), to mimic human stroke, since 5 hours is the average time from the clinical presentation of stroke to the thrombectomy procedure. A silicone coated monofilament was advanced until resistance was felt, occluding the MCA territory. At the 5 hour time point, we removed the monofilament and inserted the micoangio tubing at the same entry point on the bifurcation of the CCA. We performed variant injections using different volumes and rates, measuring delivery of dye (India Ink) and rate of induced subarachnoid hemorrhage. A rate of 4 $\mathrm{ul} / \mathrm{min}$ with a max volume of $25 \mathrm{ul}$ was optimal in males, and a rate of $2.5 \mathrm{ul} / \mathrm{min}$ with a max volume of $25 \mathrm{ul}$ was optimal in females. The results showed that, even with a large vessel occlusion, and removal of the monofilament (recanalization), the IA injection using these sex-specific rates and volumes resulted in appropriate limited dye delivery without ruptured subarachnoid hemorrhage. This IA method is ideally suited for combination with the MCAO stroke model and mirrors the human patient undergoing an endovascular thrombectomy. This model provides an investigative opportunity to test neuroprotective drugs and other pharmacotherapies.
Disclosures S. Goodwin: None. J. Roberts: None. K. Pennypacker: None. J. Fraser: None.

\section{E-018 A NOVEL 8FR ASPIRATION CATHETER SIGNIFICANTLY INCREASES THE FIRST PASS EFFECT IN COMPARISON WITH INDUSTRY STANDARD 6FR DEVICES IN AN IN-VITRO HUMAN VASCULATURE MODEL}

${ }^{1} S$ Fitzgerald*, 'D Ryan, ${ }^{2} \mathrm{~L}$ Mullins, ${ }^{3} \mathrm{~J}$ Thornton, ${ }^{4} \mathrm{R}$ Nogueira. ${ }^{1}$ Physiology, National University of Ireland Galway, Galway, IRELAND; '2Perfuze Ltd, Galway, IRELAND; ${ }^{3}$ Radiology, Beaumont Hospital Dublin, Dublin, IRELAND; ${ }^{4}$ Radiology, Grady Memorial Hospital and Emory University, Atlanta, GA

\subsection{6/neurintsurg-2020-SNIS.54}

Introduction/Purpose Achieving complete reperfusion from a single mechanical thrombectomy attempt, termed First Pass Effect (FPE), is associated with significantly improved outcomes. Increasing the lumen of aspiration catheters to $6 \mathrm{Fr}$ has previously been shown to increase the reperfusion rates in comparison to smaller lumen catheters. We evaluated the performance of a novel large bore $(0.088$ " ID) $8 \mathrm{Fr}$ aspiration catheter (Millipede 088, Perfuze Ltd.) and compared its performance against current industry standard 6Fr aspiration catheters (ACE68, Penumbra and SOFIA Plus, Microvention) in an in-vitro human vasculature model.

Methods Following National University of Ireland Research Ethics committee approval human whole blood and platelet donations were obtained from the Irish Blood Transfusion Service. Three clot analogue phenotypes representative of clots retrieved from patients were created; Red Blood Cell-Rich, Mixed and Fibrin/Platelet-Rich. Histopathological analysis was performed using Martius Scarlet Blue (MSB) staining to confirm clot composition. The in-vitro model comprised a peristaltic pump, aortic arch and circle of Willis. Flow rates and pressure were controlled to replicate in-vivo conditions. Clot analogues of each phenotype were inserted into the ICA and lodged under pulsatile flow. Clot volume was optimized to mimic the clinical scenario; $10 \mathrm{~mm}$ clots reliably lead to a Distal M1+MCA Bifurcation Occlusion and $20 \mathrm{~mm}$ clots reliably lead to an ICA-T + Proximal M1 occlusion covering both the Posterior Communicating Artery and Anterior Cerebral Artery. Five replicates of each test were performed. Endpoints were FPE and Second Pass Reperfusion Success (>90\% Retrieved).

Results Histological composition was confirmed as RBC-Rich (RBCs:92.9\%, WBCs:0.1\%, Fibrin/Platelets:7.0\%), Mixed (RBCs:79.7\%, WBCs:0.3\%, Fibrin/Platelets:20.0\%) and Fibrin/Platelet-Rich (RBCs:51.8\%, WBCs:0.3\%, Fibrin/Platelets:47.9\%). The 8Fr (Millipede 088) catheter performed better for each clot phenotype and in both occlusion locations (ICA-T \& M1) compared to the $6 \mathrm{Fr} \quad(0.068 "$ " \& $0.070 "$ ID) devices. In $10 \mathrm{~mm} \mathrm{M} 1+$ Bifurcation occlusions the $8 \mathrm{Fr}$ catheter achieved $100 \%$ FPE compared to an average of $40 \%$ in $6 \mathrm{Fr}$ devices ( $p>0.001 \%)$. In longer $20 \mathrm{~mm}$ ICA-T+Proximal M1 occlusions the Millipede 088 achieved $100 \%$ removal success within two passes in each clot phenotype compared to an average of $27 \%$ in the $6 \mathrm{Fr}$ devices $\left(\mathrm{p}>0.001^{*}\right)$. 
(A)

\section{Distal M1 + Bifurcation Occlusion}

(10mm Clot Length)
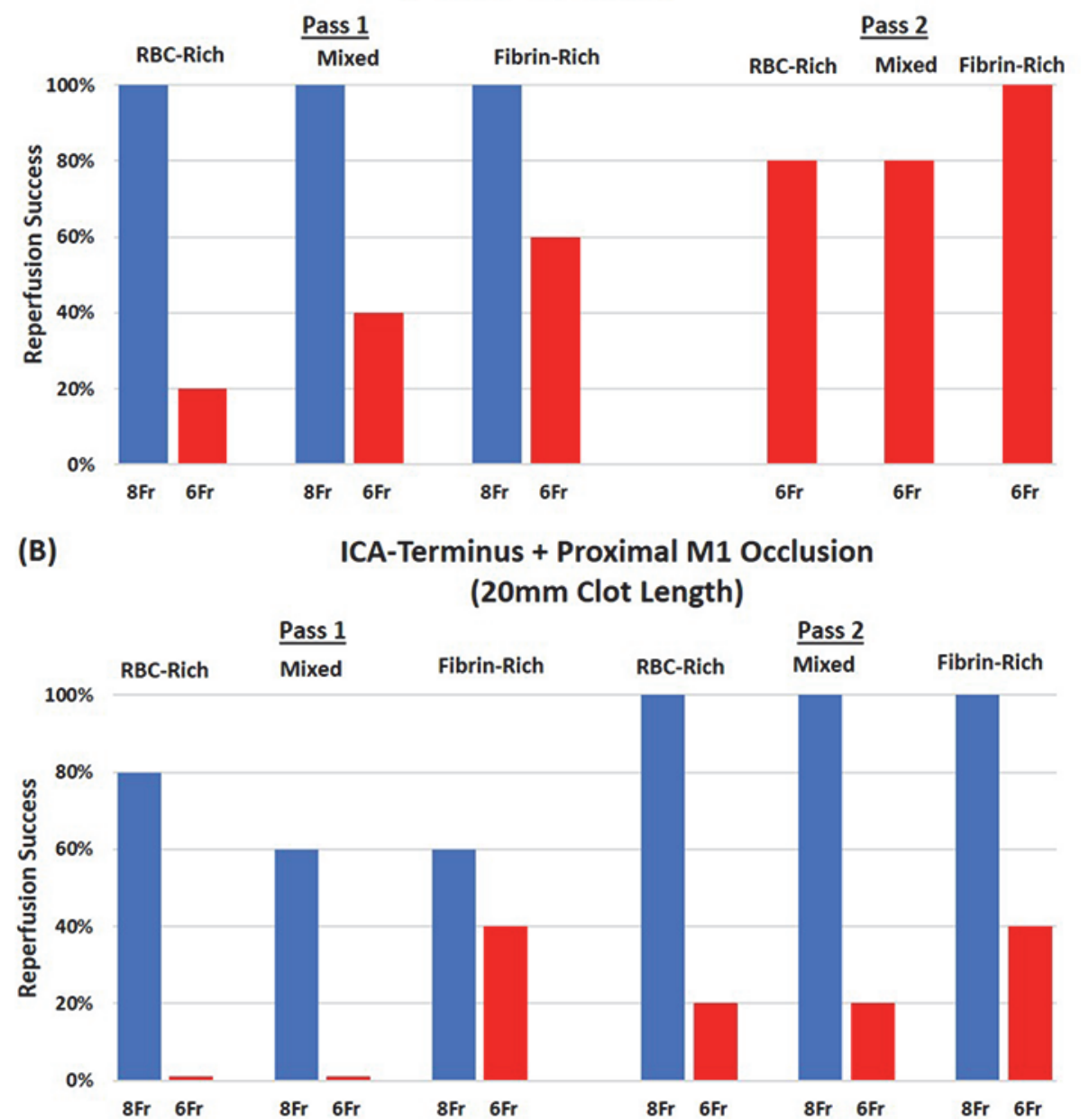

Abstract E-018 Figure 1 Comparison of the First and Second Pass Reperfusion Success Rates of the novel 8Fr Millipede 088 Catheter versus the Standard 6Fr Catheters in (A) M1+Bifurcation and (B) ICA-Terminus Occlusions. $N=5$ Replicates in all tests

Conclusions A novel 8Fr aspiration catheter demonstrates superiority over $6 \mathrm{Fr}$ aspiration catheters for each clot phenotype at the most common sites of occlusion in an in-vitro model.

Disclosures S. Fitzgerald: 1; C; Perfuze Ltd. D. Ryan: None. L. Mullins: 4; C; Perfuze Ltd. 5; C; Perfuze Ltd. J. Thornton: 2; C; Perfuze Ltd. 4; C; Perfuze Ltd. R. Nogueira: 2; C; Perfuze Ltd. 4; C; Perfuze Ltd.

\section{E-019 COMPARISON OF RABBIT MODELS OF INTRACRANIAL ATHEROSCLEROTIC DISEASE}

${ }^{1} \mathrm{M}$ Zabriskie, ${ }^{2} \mathrm{C}$ Wang, ${ }^{3} \mathrm{M}$ Alexander*. ${ }^{1}$ Radiology and Imaging Sciences, University of Utah, Salt Lake City, UT; ${ }^{2}$ Radiology, China, Shenyang, CHINA; ${ }^{3}$ Radiology and Imaging Sciences, University of Utah, Salt Lake City, UT

\subsection{6/neurintsurg-2020-SNIS.55}

Introduction/Purpose Intracranial atherosclerotic disease (ICAD) is a leading cause of ischemic stroke. Treatment controversies exist due to limited understanding of its pathophysiologic drivers. This results from a paucity of histopathological data from human lesions. Development of an animal model would allow for preclinical studies. This study seeks to characterize development of ICAD in Watanabe heritable hyperlipidemic (WHHL) and homozygous apolipoprotein E-knockout (ApoE) rabbits.

Materials and Methods Mature WHHL rabbits, mature ApoE rabbits fed a high cholesterol diet for up to six months, and juvenile wild-type New Zealand White (NZW) rabbits serving as normal controls underwent the same euthanasia protocol using perfusion fixation, with perfusate delivered through a vascular sheath inserted into a carotid artery. Animals were decapitated and the brain and intracranial arteries harvested and placed in a container with formalin. After two weeks, specimens were sliced to maximize cross-sectional orientation of the proximal intracranial arteries. Light microscopic analysis was performed by a specialized veterinary pathologist after hematoxylin and eosin staining to assess presence of ICAD. Basilar and internal carotid artery segments were each rated as having no, mild, moderate, or advanced ICAD. Two-tailed ttests were performed to compare disease prevalence among rabbit types.

Results 17 rabbits underwent evaluation, including 5 WHHL (24.3-31.6 months at euthanasia, mean $27.4 \pm 2.8), 4$ ApoE (37.8-46.8 months, mean $42.8 \pm 4.2)$, and 8 NZW rabbits (6.1-20.9 months, mean $11.1 \pm 5.2)$. In WHHL animals, 5 (33.3\%) vessel segments had ICAD on pathology (2 mild, 2 